\title{
A Prototype Wire Position Monitoring System
}

\author{
Wei Wang and Zachary Wolf \\ Metrology Department, SLAC
}

\section{INTRODUCTION ${ }^{1}$}

The Wire Position Monitoring System (WPM) will track changes in the transverse position of LCLS Beam Position Monitors (BPMs) to $1 \mu \mathrm{m}$ [1] over several weeks. This position information will be used between applications of beam based alignment to correct for changes in component alignment.

The WPM system has several requirements. The sensor range must be large enough so that precision sensor positioning is not required. The resolution needs to be small enough so that the signal can be used to monitor motion to $1 \mu \mathrm{m}$. The system must be stable enough so that system drift does not mimic motion of the component being monitored.

The WPM sensor assembly consists of two parts, the magnetic sensor and an integrated lock-in amplifier. The magnetic sensor picks up a signal from the alternating current in a stretched wire. The voltage $\mathrm{v}$ induced in the sensor is proportional to the wire displacement from the center of the sensor [2]. The integrated lock-in amplifier provides a DC output whose magnitude is proportional to the AC signal from the magnetic sensor. The DC output is either read on a digital voltmeter or digitized locally and communicated over a computer interface.

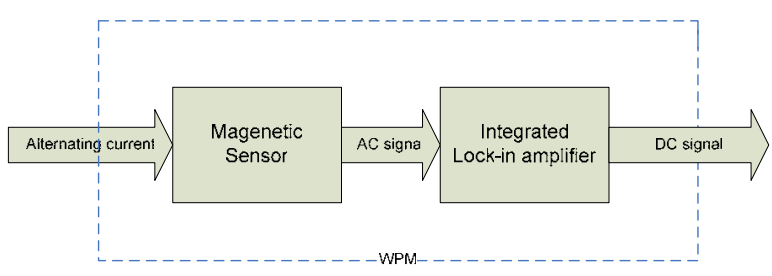

Figure 1. WPM signal flow

\section{PHASE-SENSITIVE DETECTION}

Inside the lock-in amplifier, a special rectifier, called a phase-sensitive detector (PSD), performs the AC to DC conversion and forms the heart of the electronic system. The PSD rectifies only the signal of interest while suppressing the effect of noise or interfering components which may accompany the signal.

In order to function correctly, the detector must recognize the signal of interest. This is achieved by supplying it with a reference voltage of the same frequency and with a fixed phase relationship to that of the signal. This is most commonly

${ }^{1}$ Work supported by the DOE contract DE-AC02-76SF00515. This work was performed in support of the LCLS project at SLAC. 
done by ensuring that the reference and signal are derived from the same source. In our project, the reference signal comes from half of the magnetic sensor, which is two transformer cores wired in series opposition [2]. The use of such a reference signal ensures that the system will "track" any changes in the frequency of the signal of interest, and the reference circuit is "locked" to it. This inherent tracking ability allows extremely small bandwidths to be obtained for signal-tonoise ratio improvement since there is no frequency "drift", as is the case with analog "tuned filter/rectifier” systems.

The phase-sensitive detector (PSD) is also known as a demodulator or mixer. The detector operates by multiplying two signals together, and the following analysis indicates how this gives the required outputs.

Figure 2 shows the situation where the ideal lock-in amplifier is detecting a noise-free sinusoid, identified in the diagram as "Signal In". The system is also fed with a reference signal, from which it generates an internal sinusoidal reference which is also shown in the diagram.

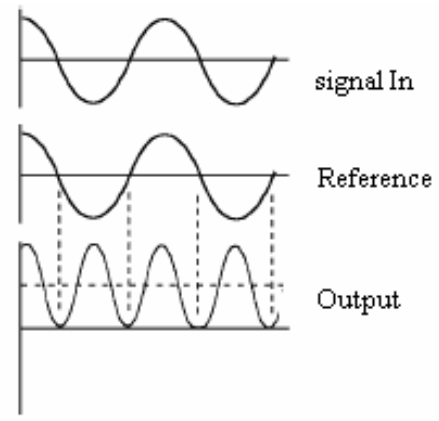

Figure 2. Ideal lock-in output

The demodulator operates by multiplying these two signals together to yield the signal identified in the diagram as "Output". Since there is no relative phase-shift between the signal and reference, the demodulator output takes the form of a sinusoid at twice the reference frequency, but with a mean, or average, level which is positive.

Figure 3 shows the same situation, except that the signal phase is now delayed by $90^{\circ}$ with respect to the reference. It can be seen that although the output still contains a signal at twice the reference frequency, the mean level is now zero.

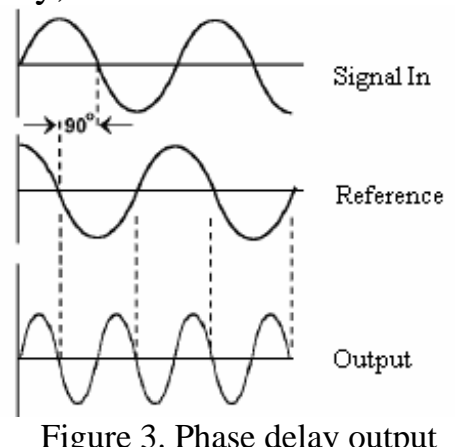

From this it can be seen that the mean level is:

- proportional to the product of the signal and reference frequency amplitudes

- related to the phase angle between the signal and reference

It will be appreciated that if the reference signal amplitude is maintained at a fixed value, and the reference phase is adjusted to ensure a relative phase-shift of zero degrees, then by measuring the mean level, the input signal amplitude can be determined. As stated before, the reference signal is from the half transformer. Small movement of the wire will not cause any noticeable change of magnitude in the reference signal, and the relative phase-shift is zero degrees. 
The mean level is the DC component of the demodulator output, so it is a relatively simple task to isolate it by using a low-pass filter.

The above discussion is based on the case of noise-free input signals, but in real applications, the signal will be accompanied by noise. This noise, which by definition has no fixed frequency or phase relationship to the reference, is also multiplied by the reference signal in the demodulator, but does not result in any change to the mean DC level. Noise components at frequencies very close to that of the reference do result in demodulator outputs at very low frequencies, but by setting the low-pass filter to a sufficiently low cut-off frequency, these can be rejected. Hence the combination of a demodulator and low-pass output filter allows signals to be measured even when accompanied by significant noise.

\section{THE LOCK-IN AMPLIFIER}

The block diagram of a typical lock-in amplifier is shown in figure 4 .

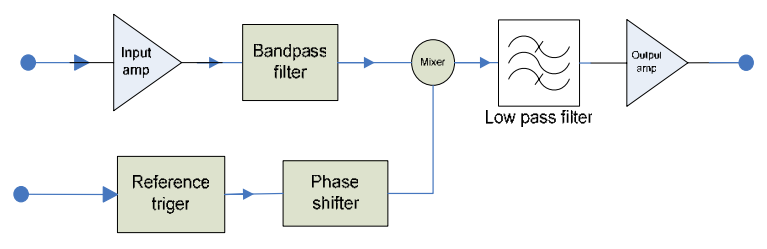

Figure 4. Typical lock-in amplifier

\section{A. SIGNAL CHANNEL}

In the signal channel the input signal, including noise, is amplified by an adjustable-gain, AC-coupled amplifier, in order to match it more closely to the optimum input signal range of the PSD. Systems are usually fitted with high impedance inputs for voltage measurements. Many also incorporate low impedance inputs for better noise matching to current sources, although in some cases the best results are obtained through the use of a separate external preamplifier.

The performance of the PSD is usually improved if the bandwidth of the noise voltages reaching it is reduced from that of the full frequency range of the system. To achieve this, the signal is passed through some form of filter, which may be simply a band rejection filter centered at the power line frequency and/or its second harmonic to reject line frequency pick-up.

\section{B. REFERENCE CHANNEL}

It has been shown that proper operation of the PSD requires the generation of a precision reference signal within the system. When a high-level, stable and noise-free reference input is provided, making lock-in measurements is a relatively simple task. However there are many instances where the available reference is far from perfect or symmetrical, and in these cases a well designed reference channel circuit is very important.

The internally generated reference is passed through a phase shifter, which is used to compensate for phase differences that may have been introduced between the signal and reference inputs by the experiment, before being applied to the PSD.

\section{MIXER}

The mixer is a multiplier. It takes the input signal and the reference and multiplies them together. When we multiply two waveforms together we get the sum and difference frequencies as the 
result. As the input signal to be measured and the reference signal are of the same frequency, the difference frequency is zero and you get a DC output which is proportional to the amplitude of the input signal and the cosine of the phase difference between the signals. By adjusting the phase of the reference signal using the reference circuit, the phase difference between the input signal and the reference can be brought to zero and hence the DC output level from the multiplier is proportional to the input signal.

\section{LOW-PASS FILTER AND OUTPUT AMPLIFIER}

The purpose of the output filter is to remove the $\mathrm{AC}$ components from the desired DC output. Practical systems employ a wide range of output filter types, implemented either as analog circuits or in digital signal processors. Usually, however, these are equivalent to one or more stages of simple single-pole "RC" type filters.

There is usually also some form of output amplifier, which may be either a DCcoupled analog circuit or a digital multiplier. The use of this amplifier, in conjunction with the input amplifier, allows the unit to handle a range of signal inputs. When there is little accompanying noise, the input amplifier can be operated at high gain without overloading the PSD, in which case little, if any, gain is needed at the output. In the case of signals buried in very large noise voltages, the reverse is the case.

The block diagram of our integrated lockin amplifier is shown in figure 5. Please check the appendix for the schematics of the PC board. The total gain is 40 ; the cutoff frequency of the low pass filter is $16.7 \mathrm{~Hz}$.

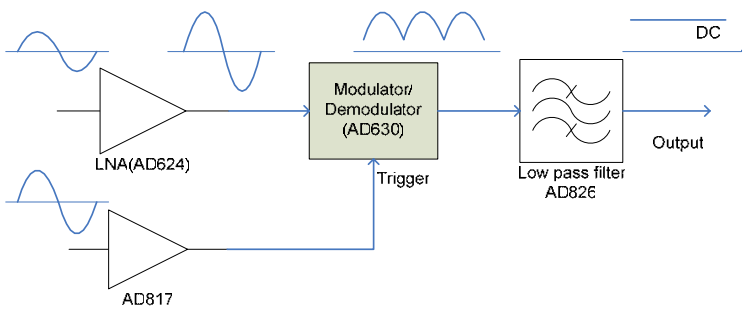

Figure 5. Integrated lock-in amplifier

\section{COMPUTER CONTROL}

The microprocessor can simplify and automate manual measurements as well as supporting remote control of the system over common computer interfaces, such as the GPIB (IEEE-488) and RS232 links. In this project, we use an HP34970 remote data acquisition system and GPIB link, and a computer program based on Lab Windows. The connection is shown as figure 6 .

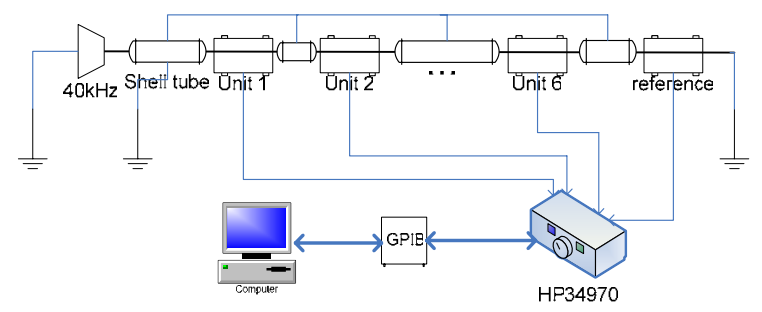

Figure 6. Layout of the measurement system

\section{MEASUREMENT}

The objective of this project is to test a prototype wire position monitoring system for possible use in the LCLS. The measurement error should be less than $1 \mu \mathrm{m}$. The wire sensor plus electronic board are mounted on the object being monitored. The input signal to the sensor is a $40 \mathrm{kHz}$ AC signal, which comes from magnetic pickup of current in the wire. The reference signal also comes from the sensor, guaranteeing the same frequency 
and a fixed phase. The output from the lock-in amplifier is a DC voltage with magnitude proportional to the AC signal. We read the data points every 2 minutes via GPIB. We debugged and tested the whole system in SLAC sector 10. The measurement units (sensors plus electronics boards) are mounted on the wall. The length of the stretched wire is $30 \mathrm{~m}$. A reference unit shares the same input as other units, but the wire is glued on the transformer on the reference unit to detect the electrical drift of our system.

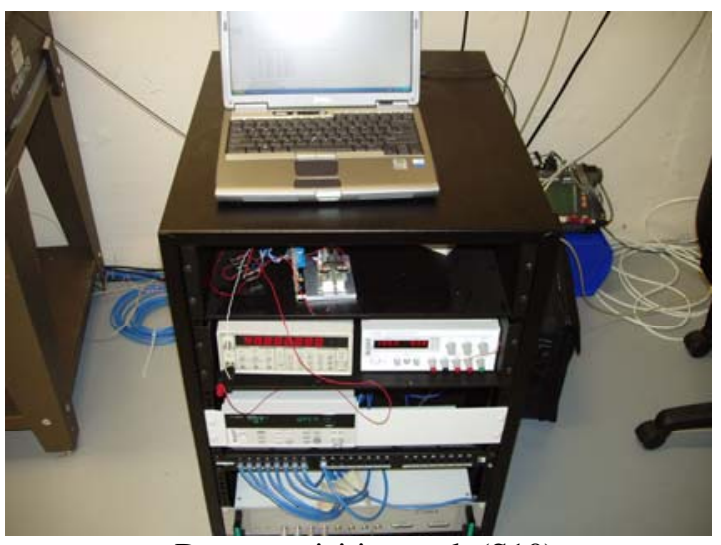

Data acquisition rack (S10)

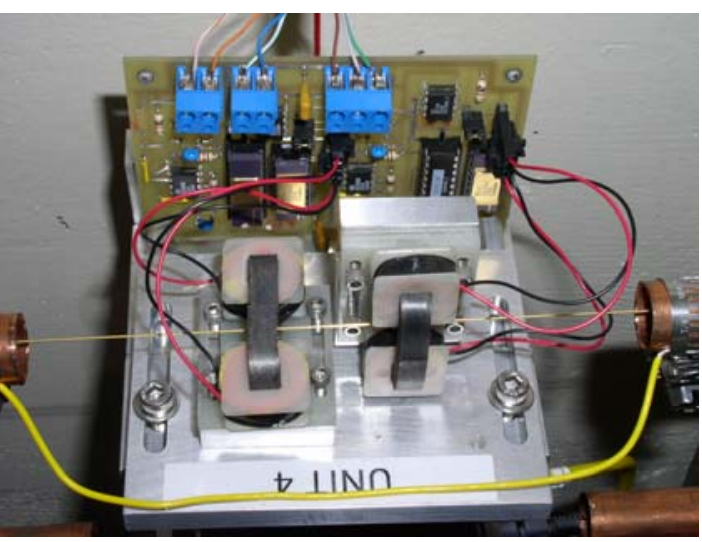

WPM sensor (S10)

Figure 7-10 show the results of sensor position over 10,000 minutes (7 days) run. As mentioned before, since the whole units are mounted on the wall, any tiny movement of the wall will cause changes of the output. We investigated other environmental factors such as temperature and airflow, especially temperature, which will affect the resistance and the length of the stretched wire. The outputs of horizontal (X) channels have several microns drift (due to wall motion) over 7 days and outputs of vertical (Y) channel have periodic changes for each day.
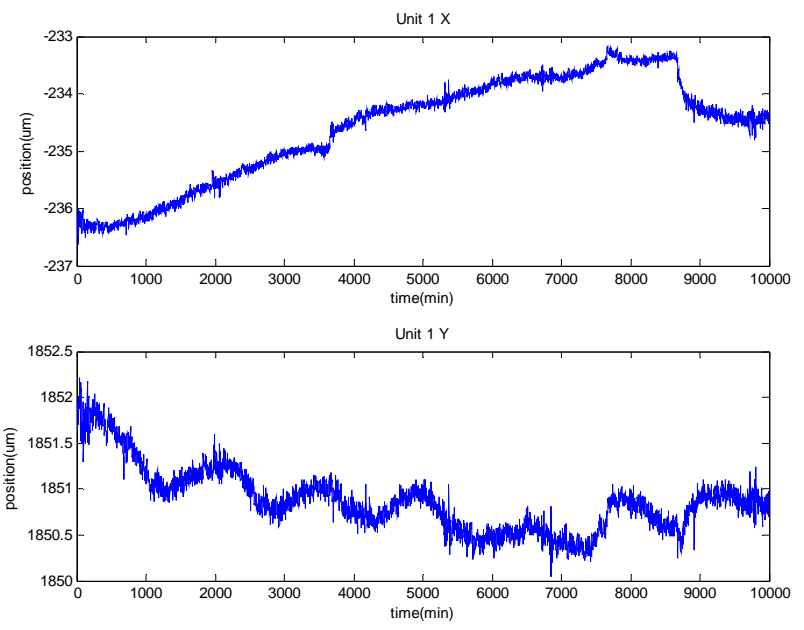

Figure 7. Unit 1 output
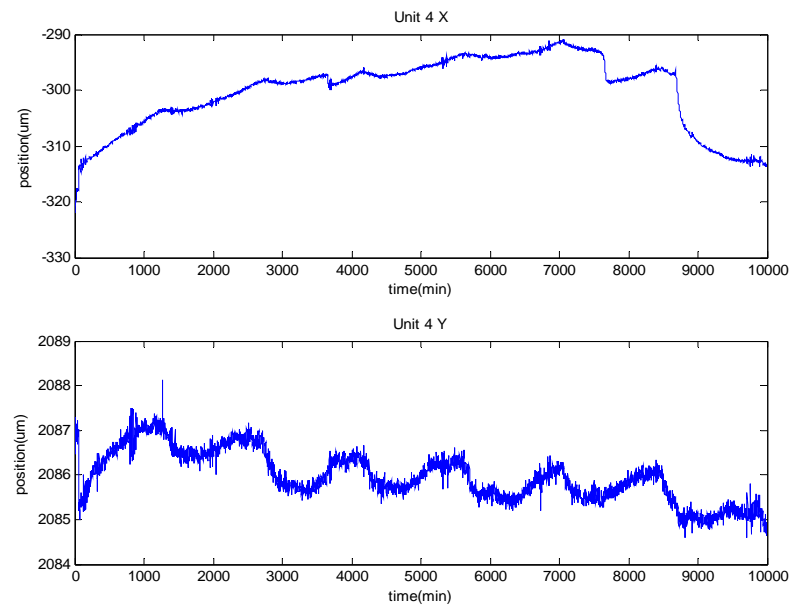

Figure 8. Unit 4 output 

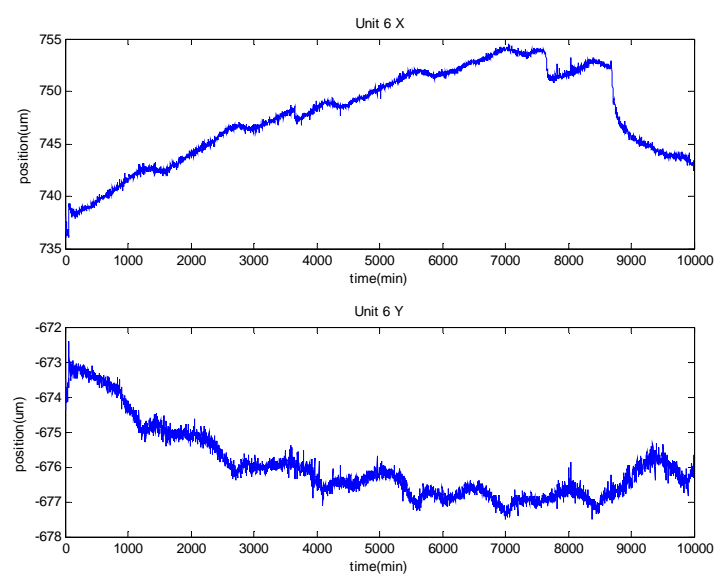

Figure 9. Unit 6 output
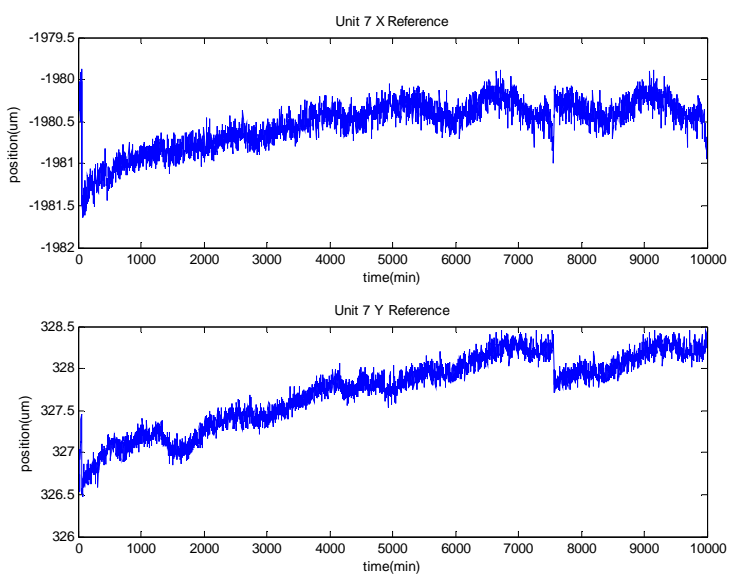

Figure 10. The reference unit output

\section{RESULTS}

The output of the reference unit tells us that the electronic drift of the system is around $1 \mu \mathrm{m}$ over 7 days. Figure 11 is the calibration data of unit $1 \mathrm{x}$ channel. As we can see, the DC output is linear with respect to the position [2]. The slope of the line is $1.7251 \mathrm{~V} / \mathrm{mm}$, which is the calibration constant in the parameter file. From the figure, we can also see that the sensor is well behaved in the range of $2 \mathrm{~mm}$. The voltage residuals from the linear regression are shown in figure 12 . We see that there exist two types of errors, systemic error and random error. The systemic error shows the (weak) nonlinearity of the linear system. After applying a quadratic fit to the residuals, we find that the biggest nonlinear offset is $3.5547 \mathrm{e}-3 \mathrm{~V}$, which tells us the systemic error due to the linear assumption is $1.993 \mu \mathrm{m}$ over $2 \mathrm{~mm}$ range. After subtracting nonlinearity error, we also find the RMS value of the residuals is $1.0518 \mathrm{e}-3 V$. Converting volts to microns, we have the random error of $0.609 \mu \mathrm{m}$, which is our estimate of the system resolution.

unit $1 x$

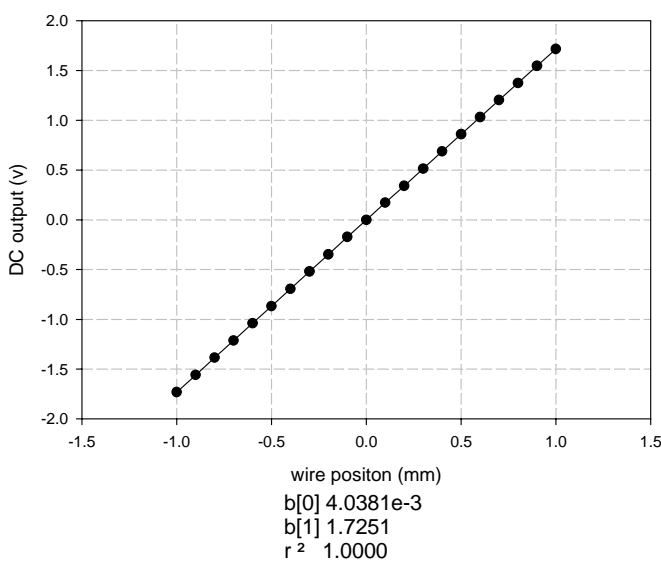

Figure 11. Unit $1 \mathrm{x}$ calibration data plot

residuals

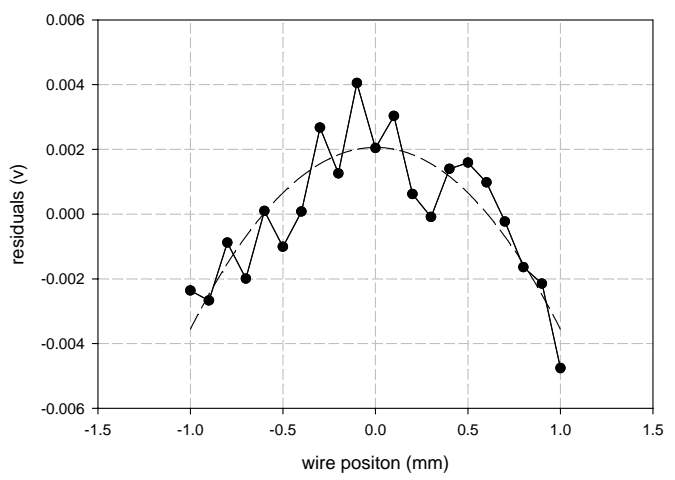

Figure 12. Voltage residuals plot

\section{KALMAN FILTER}

Consider the problem of estimating the variables of some system. In dynamic systems (that is, systems which vary with time) the system variables are often 
denoted by the term state variables. Assume that the system variables, represented by the vector $x$, are governed by the equation $x_{k+1}=A x_{k}+w_{k}$ where $w_{k}$ is random process noise, and the subscripts on the vectors represent the time step.

The Kalman filter [3] is formulated as follows. Suppose we assume that the process noise $w_{k}$ is white Gaussian noise with a covariance matrix $Q$. Further assume that the measurement noise is white Gaussian noise with a covariance matrix $R$, and that it is not correlated with the process noise. We want to formulate an estimation algorithm such that the following statistical conditions hold:

1. The expected value of our estimate is equal to the expected value of the state. That is, "on average”, our estimate of the state will equal the true state.

2. We want an estimation algorithm such that of all possible estimation algorithms, our algorithm minimizes the expected value of the square of the estimation error. That is, "on average”, our algorithm gives the "smallest" possible estimation error.

Kalman filter is the estimation algorithm which satisfies these criteria. There are many alternative ways to formulate the Kalman filter equations. One of the formulations is given in the following equations.

$$
S_{k}=P_{k}+R
$$

$$
\begin{aligned}
& K_{k}=A P_{k} S_{k}^{-1} \\
& P_{k+1}=A P_{k} A^{T}+Q-A P_{k} S_{k}^{-1} P_{k} A^{T} \\
& \hat{x}_{k+1}=A \hat{x}_{k}+K_{k}\left(z_{k+1}-A \hat{x}_{k}\right)
\end{aligned}
$$

In the above equations, the superscript -1 indicates matrix inversion and the superscript $T$ indicates matrix transposition. $S$ is the innovation covariance, $K$ is called the Kalman gain matrix, and $P$ is called the covariance of the prediction error.

The first term in equation (4) is used to derive the state estimate at time $k+1$ is just $A$ times the state estimate at time $k$. This would be the state estimate if we didn't have a measurement. In other words, the state estimate propagates in time just like the state vector. The second term in equation (4) is called the corrector term, and it represents how much to correct the propagated estimate due to our measurement. Inspection of equation (4) indicates that if the measurement noise is much greater than the process noise, $K$ will be small (that is, we won't give much credence to the measurement); if the measurement noise is much smaller than the process noise, $K$ will be large (that is, we will give a lot of credence to the measurement).

In this project, we want to measure the position which varies with time and correlate with small magnitude random noise. Figure 13 shows the estimation of position of unit 1 using Kalman filter. The result tells us that Kalman filter is capable of filtering the random noise caused by instrumental and environmental factors. 

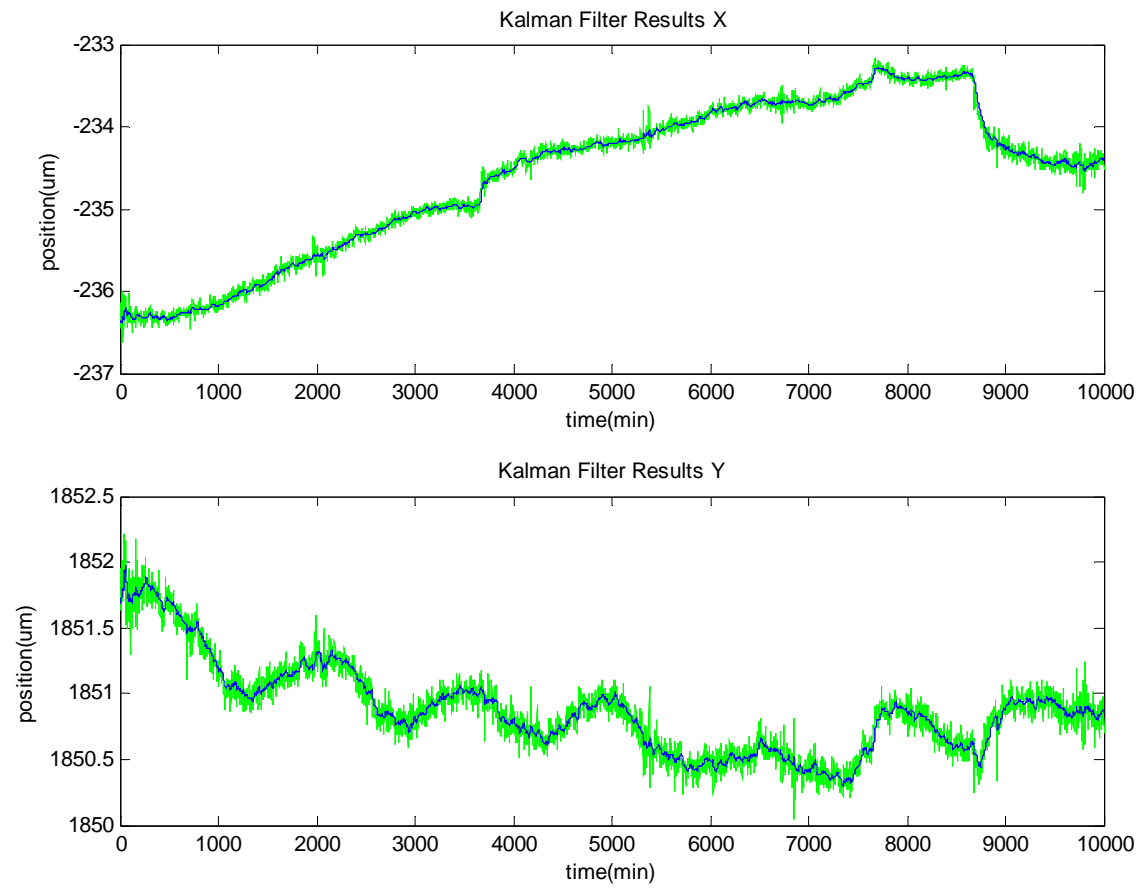

Figure 13. Kalman filter result

and Applications" Proceedings of the IEEE, Vol.63, 1975.

\section{ACKNOWLEDGEMENT}

The authors would like to thank Brendan Dix, David Jensen and Scott Jansson for their invaluable help on the mechanical design and system hardware setup.

\section{REFERENCE}

- $\quad$ [1]. LCLS Specification \#1.4-001

- $\quad$ [2]. W. K. H. Panofsky, "The Use of a Magnetic Pickup as an Alignment Indicator with a Stretched-wire Technique” TN-65-74, September 1965.

- [3]. Greg Welch and Gary Bishop, "An Introduction to the Kalman Filter," Updated April 5, 2004.

http://www.cs.unc.edu/ welch/kalman/k almanIntro.html

- [4]. Bernard Widrow and Samuel Steams, "Adaptive Signal Processing”, Prentice Hall, 1985.

- [5]. Bernard Widrow, John Glover, etc. "Adaptive Noise Cancelling: Principles 


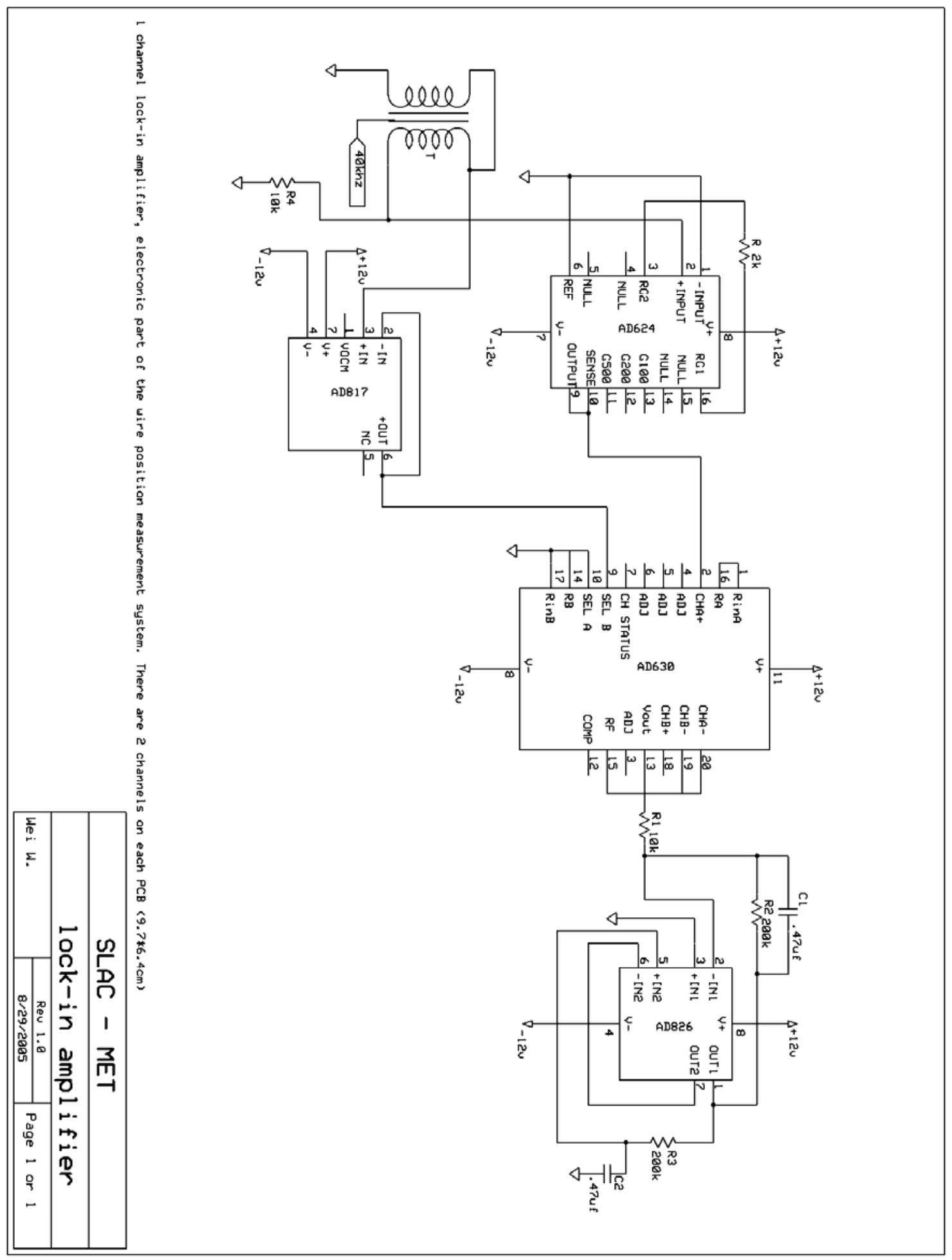

